\title{
Molecular analysis of Hsp70 mechanisms in plants and their function in response to stress
}

\begin{abstract}
Studying the strategies of improving abiotic stress tolerance is quite imperative and research under this field will increase our understanding of response mechanisms to abiotic stress such as heat. The Hsp70 is an essential regulator of protein having the tendency to maintain internal cell stability like proper folding protein and breakdown of unfolded proteins. Hsp70 holds together protein substrates to help in movement, regulation, and prevent aggregation under physical and or chemical pressure. However, this review reports the molecular mechanism of heat shock protein $70 \mathrm{kDa}$ (Hsp70) action and its structural and functional analysis, research progress on the interaction of Hsp70 with other proteins and their interaction mechanisms as well as the involvement of Hsp70 in abiotic stress responses as an adaptive defense mechanism.
\end{abstract}

Keyword: Heat shock proteins 70; Abiotic stresses; Plants; Stress mechanisms; Chaperones 